\title{
Development of Assistive Robots Using International Classification of Functioning, Disability, and Health: Concept, Applications, and Issues
}

\author{
Hideyuki Tanaka, Masahiro Yoshikawa, Eimei Oyama, \\ Yujin Wakita, and Yoshio Matsumoto \\ National Institute of Advanced Industrial Science and Technology (AIST), Japan \\ Correspondence should be addressed to Hideyuki Tanaka; hideyuki-tanaka@aist.go.jp
}

Received 20 August 2013; Accepted 17 October 2013

Academic Editor: Shigeyuki Suzuki

Copyright ( $) 2013$ Hideyuki Tanaka et al. This is an open access article distributed under the Creative Commons Attribution License, which permits unrestricted use, distribution, and reproduction in any medium, provided the original work is properly cited.

\begin{abstract}
Many assistive robots for elderly and disabled people have been developed in the past few decades. However, very few of them became commercially available. The major cause of the problem is that the cost-benefit ratio and the risk-benefit ratio of them are not good or not known. The evaluation of them should be done in the light of the impacts of assistive technologies on users' whole life, both in short-term and long-term. In this paper, we propose a framework of evaluation and design of assistive robots using ICF (International Classification of Functioning, Disability, and Health). The goal of the framework is the realization of the life design and the improvement of the quality of life using assistive technologies. We describe the concept of utilizing ICF in the development process of assistive robots, and demonstrate its utility by using some examples of practical application such as the analysis of daily living, the design of assistive robots and the evaluation of assistive robots. We also show the issues of using ICF for further development of the framework.
\end{abstract}

\section{Introduction}

There are growing needs for assistive robots which support independent life of elderly and disabled people or help people who work at the assistance and the nursing care. Although a great number of assistive robots, for example, wheelchair robots [1], walking assist robots [2], upper-limb assist robots $[3,4]$, communication robots $[5,6]$, and so forth, have been developed in the past few decades, very few of them became commercially available. We believe the major cause of the problem is that the cost-benefit (or risk-benefit) ratio is not good or not known.

Cost, risk, and benefit are three important evaluation axes of assistive robots. They are contradictory criteria, so getting full points on all the evaluation axes at the same time is difficult. Therefore, quantitative evaluation of them and the trade-off among them are indispensable.

In the case of industrial robots, the cost-benefit is comparable between the robots and the human workers, since the role of robots is simply to substitute for the human work.
Also in the case of assistive robots, we can estimate the cost, comparing the robots and the labor cost of care staffs. However, the appropriate evaluation of assistive robots is difficult unless we can estimate objectively and quantitatively the contribution of them to the improvement of QOL (quality of life). Note that the benefit of the robots here is not merely the performance as an assistive equipment, but the advantageous effect on the user and the user's life.

For the risk of the assistive robot, an international standardization of the safety of assistive robots is being promoted in a promising project "Project for Practical Application of Service Robots" (NEDO, FY2009-2013) [7]. However, the "risk" of being dealt with in this project is limited to the safety issues of mechanical/electrical aspects of the hardware. Other risks caused by use of assistive robots are not considered, for example, the risk of a disuse syndrome [8] which is the reduction of activities derived from excessive assistance by robots.

The evaluation of cost-benefit and risk-benefit should be done in the light of the impacts of assistive technologies on 
users' whole life, both in short-term and long-term. This needs a sharable and exhaustive description language for robot and human, which covers a wide range of human life. International Classification of Functioning, Disability, and Health (ICF) [9] which was approved by the World Health Organization (WHO) in 2001 is suitable for this purpose. In this paper, we propose a framework of development of assistive robots using ICF, which includes the evaluation of the assistive technologies in users' life.

The rest of the paper is organized as follows. Section 2 describes the related conventional works on describing functions of a robot and a person. ICF is then introduced as a new description language. In Section 3, the concept of how to apply ICF to the development of assistive robots is described. We show some examples of the application of the concept from our studies. Based on these experiences, in Section 4, we describe the issues of ICF towards its advanced usage in engineering systems.

\section{Related Works}

2.1. Classification of Behaviors of Human and Robots. Description of behavior of human and robots in a common way is a key concept in our study. This section describes the related works from this point of view [11].

Many frameworks for standardization of agents including robots have been proposed as follows:

(i) RAC (Robot Action Commands) [15],

(ii) RSi (Robot Service Initiative) Protocol [16],

(iii) BML (Behavior Markup Language).

RAC is a set of generalized motion commands defined as an extension of ORiN (Open Robot Interface for the Network) [17] which operates on different robotic platforms such as industrial robots and pet robots. RSi Protocol is a communication protocol specification for robotic services that uses the network. It promotes realization of service robots which provide physical services and information services at home and in the office. BML is designed to be a general mark-up language to express multimodal behavior of agents. It provides a framework for defining multimodal gestures (including the face, the head, the hands, and the body) and defining timing-synchronization. The language is to describe behavior in an abstract way, independent of the agent model or the actual engine realizing the behavior. Although these three description methods were invented in order to generalize and standardize the behaviors of robots independent of the platforms, they seem to have something to do with the description of the specification of an assistive robot as well as the needs of a person to be assisted.

Meanwhile, several methods for describing human activities are proposed and utilized mainly in the medical and healthcare fields as follows:

(i) MET (Metabolic Equivalent of Task),

(ii) ADL (Activities of Daily Living),

(iii) IADL (Instrumental Activities of Daily Living), (iv) ICF (International Classification of Functioning, Disability and Health).

MET is a unit used to compare the working metabolic rate (the amount of oxygen used by the body during physical activity) to the resting metabolic rate [18]. It is a way to compare the amount of exertion required for different activities. At rest, the body uses one MET for basic functions such as breathing. Moderate physical activity requires 3 to $6 \mathrm{METs}$, and vigorous physical activity requires more than 6 METs.

ADLs represent the things we normally do in daily living including any daily activities we perform for self-care (such as feeding ourselves, bathing, dressing, and grooming), work, homemaking, and leisure. The ability or inability to perform ADLs can be used as a very practical measure of ability/disability in many disorders [3]. Health professionals refer to the ability or inability to perform ADLs as a measurement of the functional status of a person. This measurement is useful for assessing the elderly, the mentally ill, and those with chronic diseases to evaluate what type of health care services an individual may need.

IADL stands for six daily tasks (light housework, preparing meals, taking medications, shopping for groceries or clothes, using the telephone, and managing money) that enable the patient to live independently in the community.

These three description methods can indicate how actively a person is living and what type of assistance a person may need in some aspects.

However, they are rather rough in finding out the detailed needs of a person. ICF is another way of describing the activities of an individual with finer and wider description capability, which is explained in Section 3.

2.2. ICF: International Classification of Functioning, Disability, and Health. International Classification of Functioning, Disability and Health, known as ICF, is a classification of health and health-related components. It was approved by the World Health Organization (WHO). ICF belongs to the Family of International Classifications developed by the $\mathrm{WHO}$ (WHO-FIC) and constitutes the core classification together with ICD-10 (International Classification of Diseases, Tenthrevision) which classifies the health conditions (diseases, disorders injuries, etc.). ICF classifies functioning and disability associated with health conditions. Therefore, ICF and the ICD-10 are complementary.

The aim of ICF is to provide a unified and standard language and framework for the description of health and health-related states.

2.2.1. Classification. ICF has two parts, each with two components:

Part 1. Functioning and Disability:

(a) body Functions and Structures,

(b) activities and Participation.

Part 2. Contextual Factors:

(a) environmental factors,

(b) personal factors. 

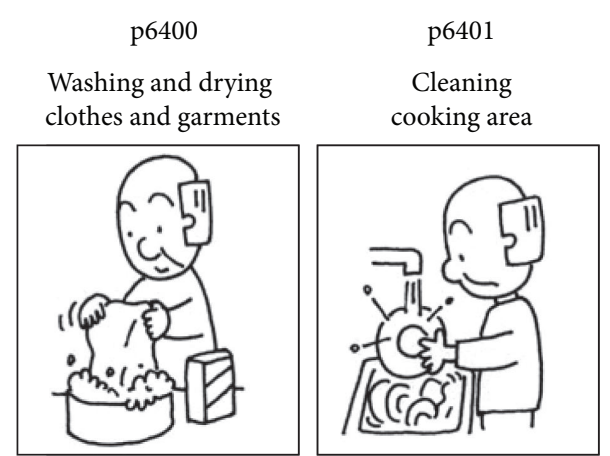

(a)
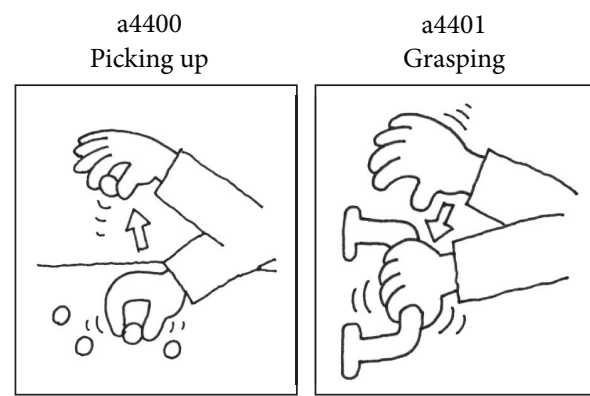

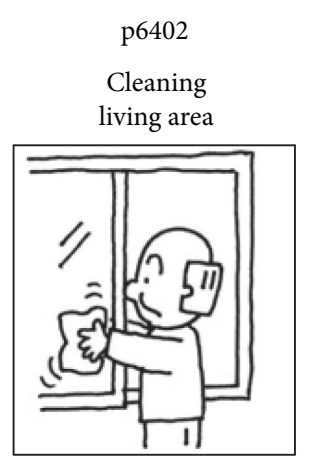

(a)
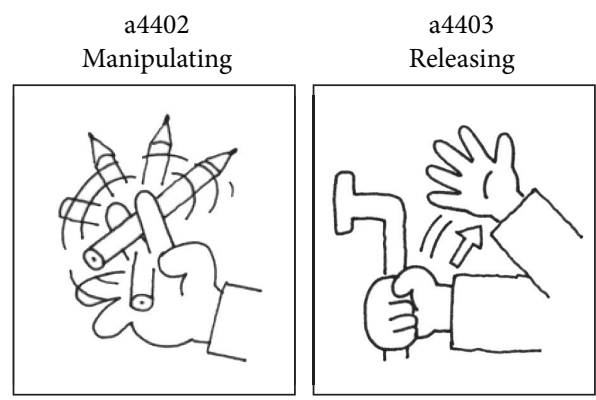

(b)

FIGURE 1: Examples of participation-level (a) and task-level (b) activities and corresponding codes [10].

Each component consists of various domains. Each domain consists of categories, which are the units of classification. For example, "Activities and Participation" component is further divided into categories shown below.
a1: Learning and applying knowledge,
a2: general tasks and demands,
a3: communication,
a4: mobility,
a5: self-care,
a6/p6: domestic life,
a7/p7: interpersonal interactions and relationships,
a8/p8: major life areas,
a9/p9: community, and social and civic life.

The ICF categories are coded by a character and a numeric code that starts with the chapter number (one digit), followed by the second level (two digits), and the third and fourth levels (one digit each). The characters "a" and "p" in the above list denote task-level activity (Activities) and participationlevel activity (Participation), respectively (Figure 1). Also, the categories are "nested" so that broader categories are defined to include more detailed subcategories of the parent category. For instance, a detailed basic motion "carrying in the hands" is classified into a subcategory of "a4: mobility" as shown below.

\section{a4: Mobility:}

a410: changing basic body position, a415: maintaining a body position, a420: transferring oneself, a429: changing and maintaining body position, other specified and unspecified, a430: lifting and carrying objects:

a4300: lifting,

a4301: carrying in the hands, a4302: carrying in the arms, a4303: carrying on shoulders, hip, and back,

a4304: carrying on the head, a4305: putting down objects, a4308: lifting and carrying, other specified, a4309: lifting and carrying, unspecified.

1,424 functions and factors are coded in ICF. When we record the health and health-related states of an individual, we select the appropriate category codes and then add qualifiers, which are numeric codes that specify the extent or the magnitude of the functioning or disability in that category, or the extent to which an environmental factor is a facilitator or barrier.

2.2.2. Model of Human Life. In addition to the classification, ICF provides a holistic model which grasps the entire picture of human life. Figure 2 shows the model (we call it "ICF model"). In this model, various components of ICF interact with each other. An individual's functioning in a specific domain is an interaction or complex relationship between the health condition and contextual factors (i.e., environmental 


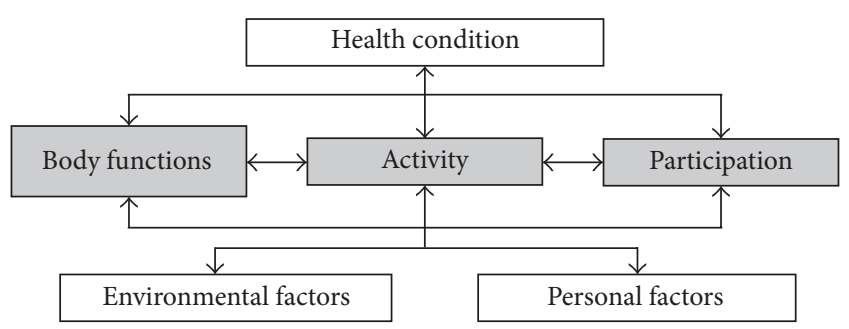

FIgURe 2: ICF model.

and personal factors). ICF is based on an integration of the medical model and the social model. Assistive robots belong to the "environmental factor" and have impacts on the functioning components as this model expresses.

The favorable features of ICF for our purpose are summarized as follows:

(1) comprehensive classification to describe human life,

(2) standardized language (framework) which is sharable among people involved,

(3) holistic perspective (model) about human life.

\section{Development of Assistive Robots Using ICF}

3.1. Problem of Evaluation of Assistive Robots. Conventional researches of assistive robots tended to follow "seeds-oriented approach," in which a robot is produced as an application of the preexisting seeds (technologies), and they seem to have paid few attention on how they bring benefits to users' life. As we mentioned in Section 1, the benefit here is not merely the physical performance of the robots.

The evaluation of assistive robots in previous studies tended to focus on medical/functional ones [4, 19], which measure the execution time and the success rate of a few single tasks, for example. However, we have questions about such evaluations, from the viewpoint of true contribution of assistive robots. Is it a true need that a user performs a single task using an assistive robot? Does the robot bring benefits to the user's life that more than compensate for the cost, risk, and the other side effects? Does the high performance robot have possibility of being a cause of the disuse syndrome?

We believe that we cannot judge whether a function meets the true needs and whether it has no problem in daily living, unless we conduct proving tests and clinical evaluations adapted to each user's life. In the existing conditions, the benefit evaluation of assistive robots is insufficient. We recognize the following two problems.

(1) Most of the proving tests of assistive robots put emphasis on the questionnaires about the feeling of the effects and do not investigate the benefits to the users' life by quantitative and statistic evaluation index.

(2) The contents of the proving tests are very small elements (actions) of the users' daily living. The relation between these actions and the improvement of users' life is unclear.
3.2. Assistive Robots in ICF. As described in Section 2.2, ICF has three factors, that is, health conditions, environmental factors, and personal factors, which interact with the functioning of a person. An assistive robot is defined as an element of the environmental factors. In concrete terms, it corresponds to the following:

(i) e1151: assistive products and technology for personal use in daily living,

(ii) e1201: assistive products and technology for personal indoor and outdoor mobility and transportation.

In the ICF model (Figure 2), we see that the assistive robots

(1) not only support "Activity" but also have impacts on "Body functions and structure" and "Participation,"

(2) have impacts on the functioning of human, by combining with other environmental factors, for example, welfare equipment, welfare services, and housing environment.

As for (1), for example, we can recognize that an assistive robotic arm has impacts on not only "a4300: lifting" (a subject of direct supporting) but also "p820: school education" and "p920: recreation and leisure".

We should also realize that there are negative impacts in addition to the positive impacts. A typical example of the negative impact is the disuse syndrome. Robot developers tend to think that higher robot performance makes a better assistance. However, if a robot technology substitutes for human activities and the user stops doing an activity which can be done originally by the user, it is an excessive assistance which leads to the disuse syndrome. Thus, clear definition of function of assistive robot is necessary for determining "indication and contraindication" (who should utilize and who should not utilize) of assistive robots.

As for (2), it is no wonder that a single assistive robot cannot be the solution to all the problem, because the users live in the various environmental factors, for example, living environment, personal relationships, social services, and sociocultural influences. We need to design the combinational usage of the assistive robots with existing welfare equipment (wheelchairs, prosthetic limbs, walking sticks, care lifts, etc.) for effective assistance. Also, reorganization of the environment for the usage of robots by house renovation and cooperation with other social services are important matters to be considered.

3.3. Concept of Life Design Using ICF. The rest of this section describes the ideas on how to utilize ICF in the development process of assistive robots-analyzing and discovering needs in daily lives, designing robots, and evaluating the products. Figure 3 shows the concept of life design with various assistive methods based on ICF. If the status of current life and desired life are described using ICF, and the effect of various assistive methods is defined by ICF, the combinational usage of assistive methods to fill the gap will be analytically obtained.

There are many ways to fill in the gap as follows:

(i) functional training, 


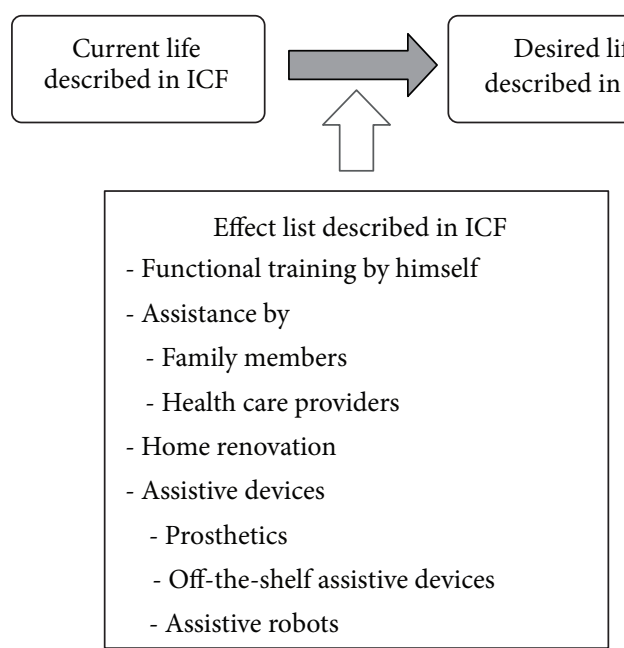

FIGURE 3: Concept of life design based on ICF [11].

(ii) support from family,

(iii) support from home helpers,

(iv) house renovations,

(v) conventional assistive devices,

(vi) assistive robots.

The robotics researchers and developers should notice that robotics technology is not the only way to realize the assistance, but the summation of the above-mentioned ways is the assistance.

In this design framework, we place great importance on discrimination of "function" and "motion/behavior" and relation between them. A function is a concept which emerges when a sequence of motions/behaviors has a "purpose (or goal)" [20]. For example, an alternating motion of legs becomes a function of moving around when a human has a certain purpose. An important finding is that the way to achieve a function is not unique. Therefore, we can plan various ways to support the functions in daily livings, and it means that we can absorb the individual differences of motions to achieve a function, because the aim of the assistance is not to recover all motions/behaviors of a human, but to recover the functions a human wants to achieve.

It should also be noticed that the less functions and motions an assistive robot has, the less cost and time is necessary to realize it. We should not try to develop an all-round assistive robot. Instead, we should analyze the daily livings of a person and develop simple assistive robots or devices with truly necessary and sufficient functions or motions for the person, in combination with other ways mentioned above.

In the following, we show our practical examples of the application of ICF in developing assistive robots, such as analysis of functions in daily livings (see Section 3.4) and robot design and evaluation of assistive robots (see Section 3.5).

3.4. Analysis of Daily Living and Design of Assistive Robots. It is important to analyze the functions conducted in our daily livings in order to understand the structure of the life, because it is not clear what kinds of task-level activities should be assisted for achieving a certain participation-level activity. Supposing that we need to build a cooking assistant robot for a handicapped person, then the concrete contents of assistance as well as the specification of a robot should be determined first. It is actually very difficult to achieve in an objective and quantitative manner. In addition, it should be also noted that excess support of assistive robots may result in disuse syndrome. Too much spoils, too little does not satisfy. Therefore, the design of assistance should be performed to avoid the excess and the deficiency.

3.4.1. Reasonable Design of Assistive Robots Based on Real Life Data. We recorded a life-log of a healthy person and analyzed it based on the ICF [11]. Action, time, duration, place, target object, and purpose were recorded by voice recorder every time when an action was taken, and 3964 activities were recorded for five days. Figure 4 indicates the histogram of all recorded activities. It can be seen that "pick and place," which are the very basic motion of the robot studied for decades, are the most frequent activities performed in the daily lives. Figure 5 indicates the task-level activities that "cooking" includes.

By analyzing this life-log data, it was found out that "lifting (a4300)" is included in 43\% of participation-level behaviors. By further analyzing them, it was found out that approximately $90 \%$ of the objects of "lifting (a4300)" activities were less than $300 \mathrm{~g}$. The exceptions were, for instance, a kettle filled with water $(1 \mathrm{~kg})$ for "preparing complex meals (p6301)" and a vacuum cleaner (3.8 kg) for "cleaning (p6402)." This kind of information is very useful for finding out the needs and designing assistive robots based on the evidence of the real life. If a robot is able to lift up an object which weights $300 \mathrm{~g}$, it can cover $90 \%$ of daily activities. The remaining $10 \%$ of objects can be handled by combinational usage of other equipment, such as an electric pot with water supply function instead of bringing a kettle, and a robot cleaner instead of using a vacuum cleaner. This idea prevents us from developing excessively high performance, heavy, and expensive robots. Thus, we can efficiently and reasonably design assistive robots based on life analysis using ICF.

3.4.2. Adaptive Design of Assistive Robots to Users. We investigated the usage of both hands in "p630: cooking" of daily life [12]. We recorded videos of cooking scene with a head-mounted camera and analyzed the usage of each hand in terms of the frequency and the time of actions. The analysis was conducted using a newly developed videotagging tool (Figure 6). We can record the action and its time at the same time, by selecting the on-going action from the left-side column while we are playing the video. The actions are classified based on ICF. Figure 7 shows a result of analysis.

We confirmed that the usage of the nondominant hand is auxiliary and greatly different from that of the dominant hand. In concrete terms, we found the following facts. 


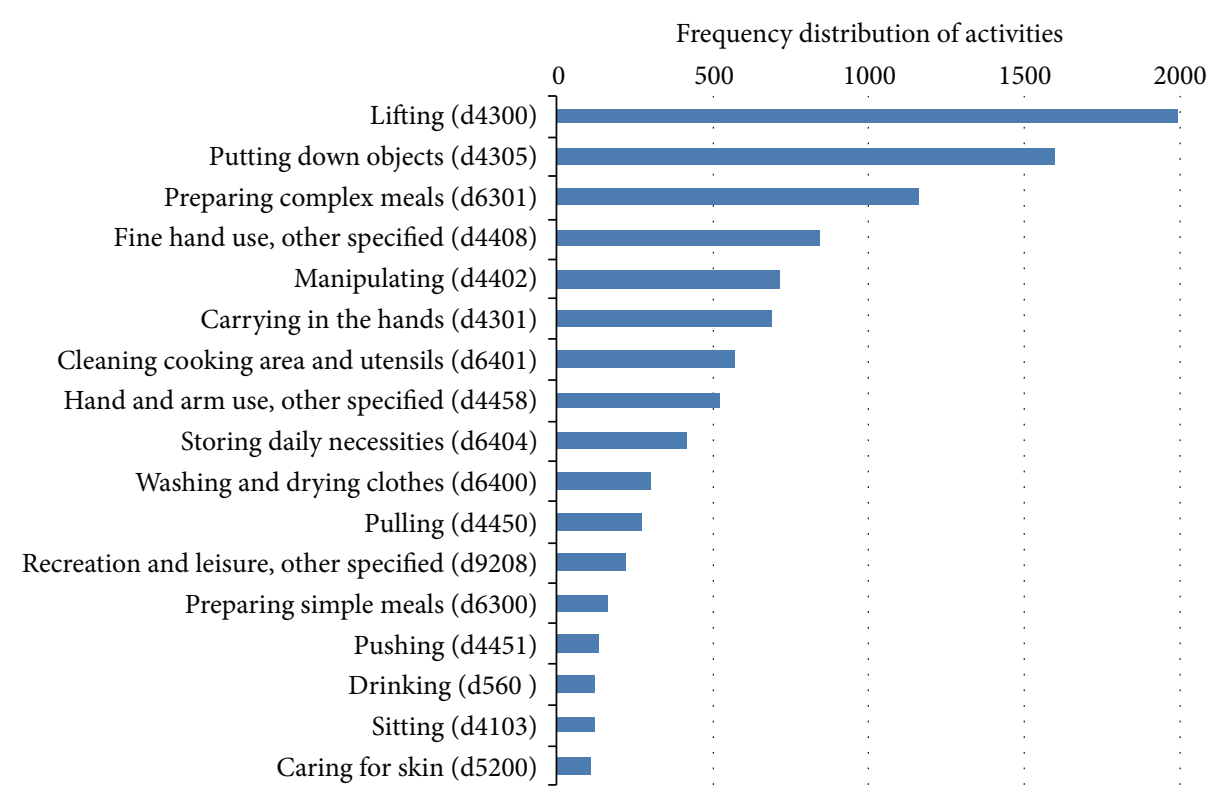

FIGURE 4: Frequency of distribution of activities [11].

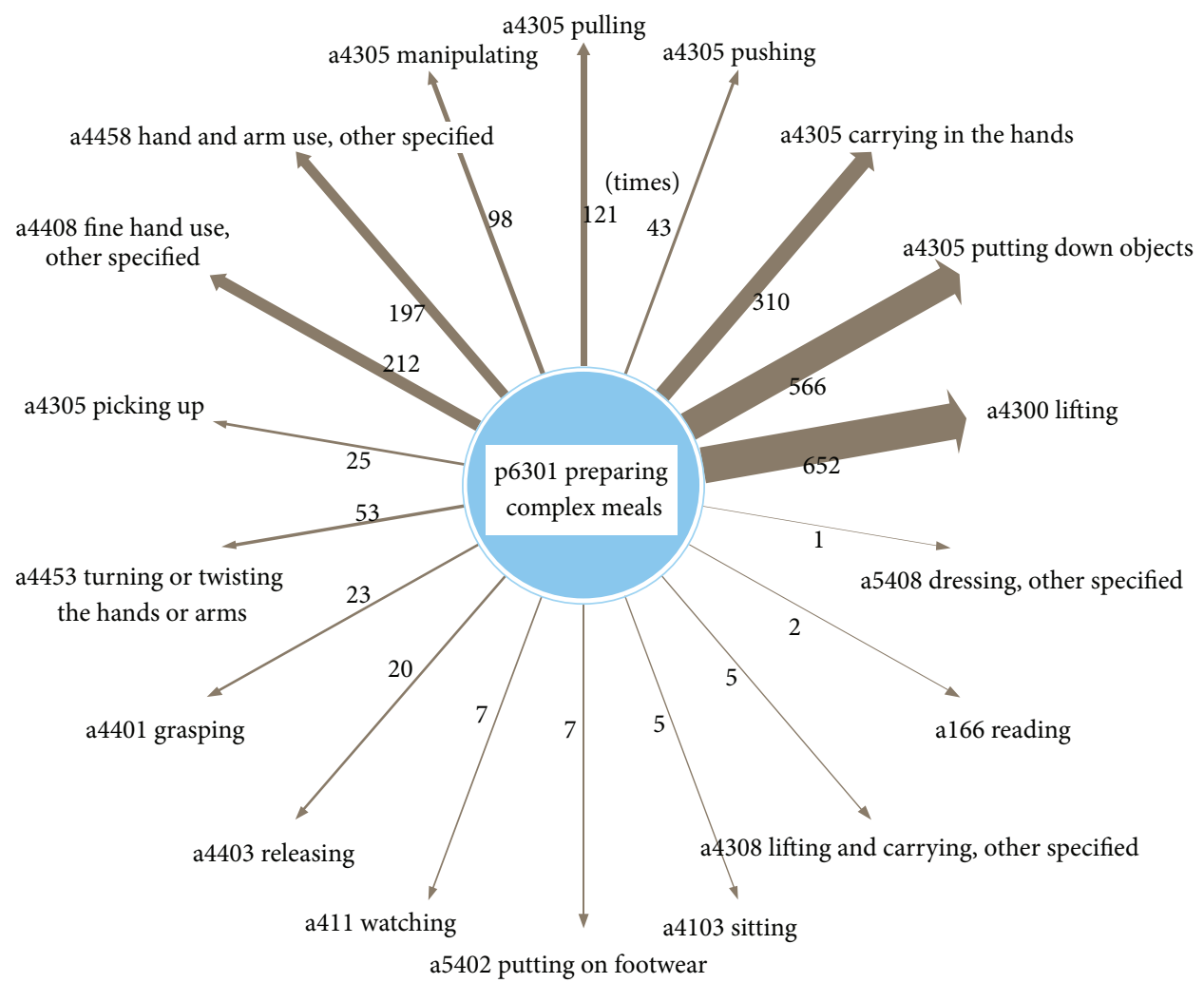

FIGURE 5: Activities included related with "preparing complex meals" [11].

(1) "Manipulating (a4402)" and "turning or twisting the hands or arms (a4453)" are performed only with dominant hand.

(2) "Grasping (a4401)" and "pushing (a4451)" are performed mostly only with nondominant hand.
(3) $83 \%$ of actions in cooking consist of only four actions such as "lifting and carrying objects (a430)," "pushing (a4451)," "picking up (a4400)," and "grasping (a4401)."

These results revealed that assistive devices for nondominant hand do not need the "twist" motion in cooking, and 


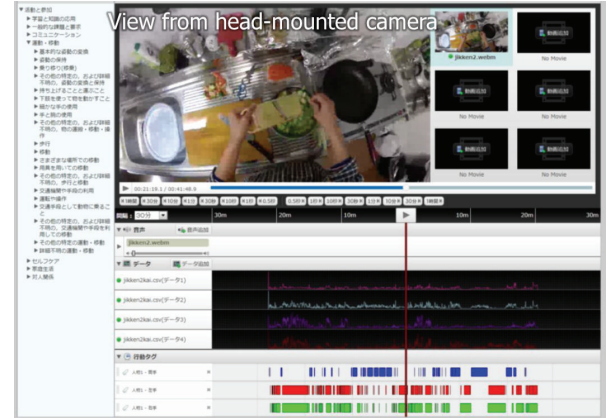

Figure 6: Analysis using video-tagging tool and ICF [12].

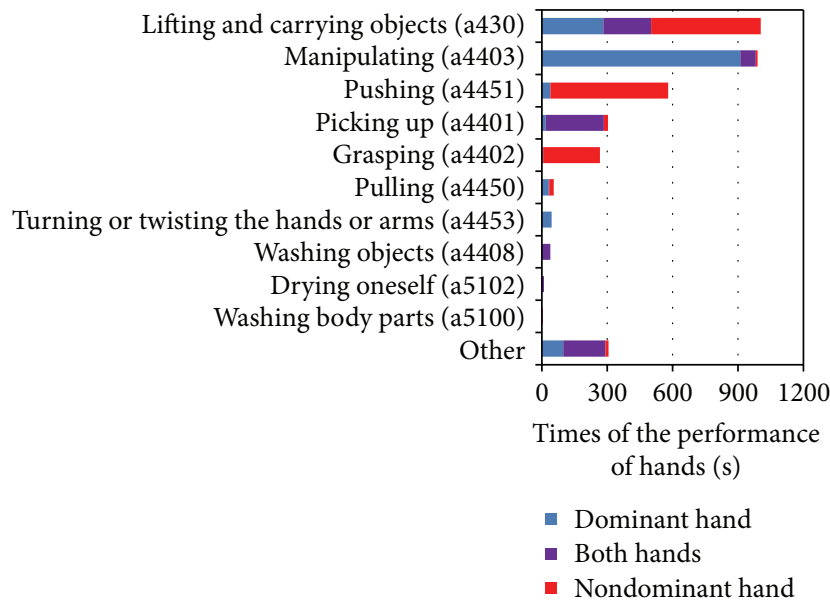

FIGURE 7: Time of actions for each hand in cooking [12].

we can simplify the structure of the assistive devices for nondominant hand by excluding one degree of freedom. This leads to the development of simple, light weight, low-cost, but useful assistive devices/robots, which adapt to the utilization form of each user.

3.5. Evaluation of Assistive Robots. In this section, we show some results of evaluation of assistive robotic arms using ICF [13]. The subjects are iARM [21] and RAPUDA [22] (Figure 8).

3.5.1. Qualitative Functionality Evaluation of Assistive Arms. In order to indicate the objective performance of an assistive robot, it should have information including following items:
(A) tasks that the robot can perform,
(B) objects that the robot can handle,
(C) environments where the robot can work,
(D) indication and contraindication of users.

ICF can be utilized for describing (A) and (D). The qualitative and quantitative description of the specification of a robot is especially important when comparing a robot with another. The iARM is an off-the-shelf robotic arm from Exact Dynamics, and the RAPUDA (Robotic Arm for Persons with
Upper-limb disAbilities) is a novel robotic arm with linear mechanism developed at AIST. By utilizing the RAPUDA, we have demonstrated that a bottle of water can be picked up from a table and taken to the mouth using a simple joystick or single-switch interfaces [23]. However, the representation of the specification such as "RAPUDA is capable of bringing a bottle of water from a table to a mouth. Maximum weight is $0.5 \mathrm{~kg}$ " is not sufficient. This is because a potential user cannot know the difference between the two robots as to which tasks can be performed and which tasks cannot. It is further difficult to imagine how the whole life will change when a user introduces the robot in his/her daily living.

There has been efforts in the community to determine a "benchmark task" for assistive robots $[24,25]$. It will also be beneficial for a user to clearly indicate what activities can be performed in terms of ICF. As a preliminary trial, we described the tasks that the assistive robotic arms can perform. Both the iARM and the RAPUDA can perform following tasks:

(i) a430: lifting and carrying object:
(a) a4300: lifting,
(b) a4301: carrying in the hands,
(c) a4305: putting down objects,

(ii) a440: fine hand use:
(a) a4400: picking up,
(b) a4401: grasping,
(c) a4402: manipulating,
(d) a4403: releasing,

(iii) a445: hand and arm use:
(a) a4450: pulling,
(b) a4451: pushing,
(c) a4452: reaching,
(d) a4453: turning or twisting in the hands or arms.

On the other hand, both robotic arms are not capable of performing following tasks due to the velocity limitation:

a445: hand and arm use:
(a) a4454: throwing,
(b) a4455: catching.

This kind of information corresponds to (A) tasks that the robot can perform. We show other usage of ICF related to (B), (C), and (D) in the following sections.

\subsubsection{Quantitative Functionality Evaluation of Assistive Arms.} Young et al. [24] presented a prioritized list of 43 objects for Amyotrophic Lateral Sclerosis (ALS) patients to pick up using a robotic arm. We used these 43 objects as target objects, and conducted operation experiments about "a4300: lifting" by the iARM and the RAPUDA. Figure 9 shows the scenes of the experiments. We put an object on a table and repeated 


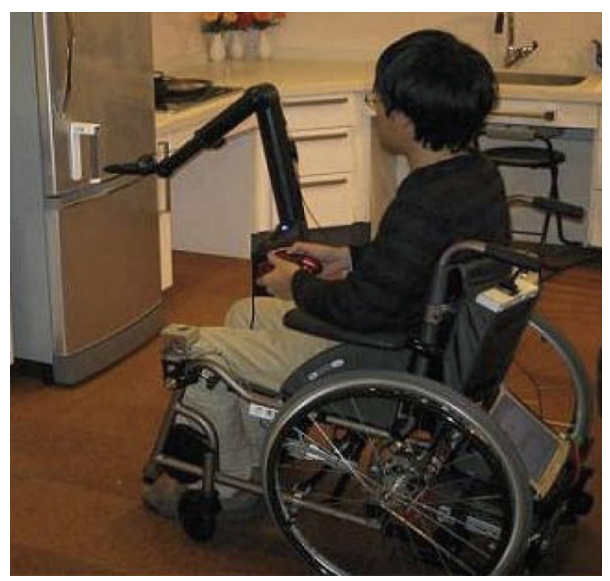

(a)

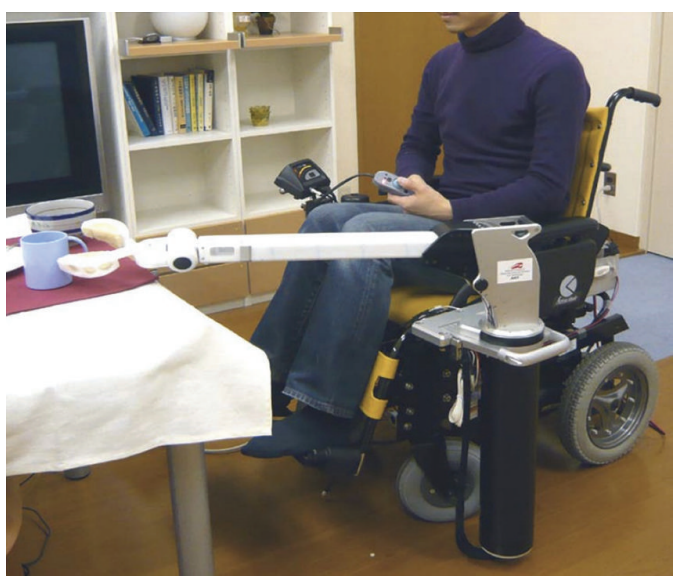

(b)

FIGURE 8: Assistive robotic arms: iARM (a) and RAPUDA (b).

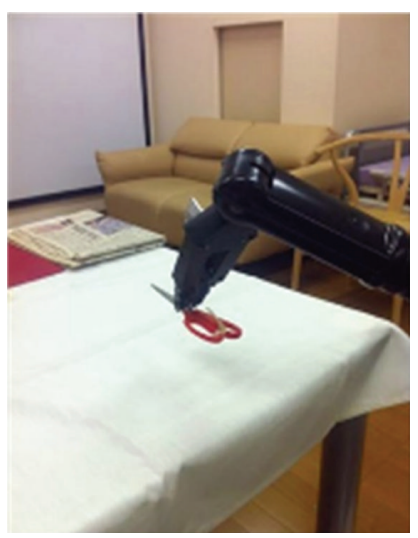

(a)

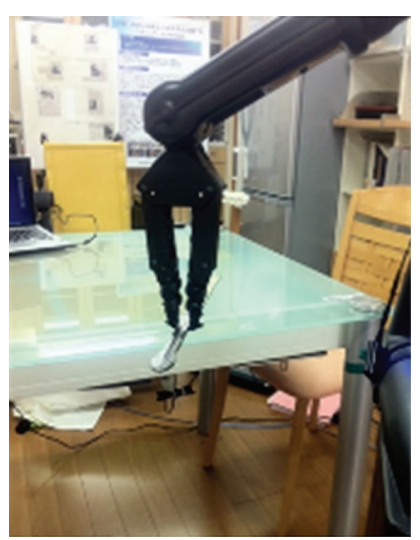

(b)

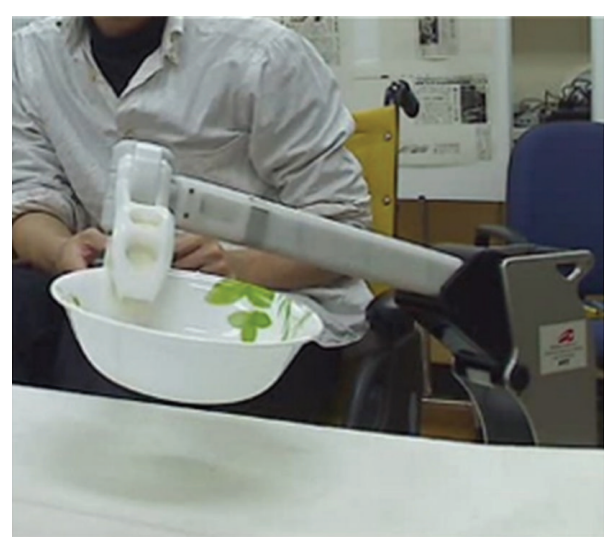

(c)

FIGURE 9: Evaluation experiment of assistive robotic arms [13].

trials of lifting up the object, in a given period of time, then recorded the success rate.

The success rate varies depending on the conditions such as the environment and grasping method. As the environment condition, we set presence/absence of a tablecloth (TC), which changes the slippage of the target object. As the grasping method, we set use/nonuse of table-edge (TE). When we use the table-edge, we are allowed to grasp an object after sliding it to the table-edge, so that a portion of the object is out of the table. In addition, the presence/absence of rubber finger (RF) is another condition in the case of the iARM. The standard conditions are presence of TC, use of TE, and presence of RF. The result is shown in Table 1.

The difference of the success rate between the iARM and the RAPUDA depends on mostly the hand (not the arm). Because the hand (finger) of the RAPUDA is thicker than that of the iARM, the RAPUDA is not good at grasping flat and thin objects such as books and magazines. When it did not use the table-edge, table knife, straw, plastic container, newspaper, wallet, coin, and stick were hard to lift.
Such information corresponds to (B) objects that the robot can handle and $(C)$ environments where the robot can work, which is important to clarify the performance of assistive robots in practical usage.

3.5.3. Requirement for Users. Adequate operation is required to the users of assistive robots. This includes the operation of the user interface, judgment of dangers, and finding of the errors of robot. We listed up the requirements for the user of assistive robotic arms as follows:

(i) b130: energy and drive functions (for keeping goal of task),

(ii) b140: attention functions (for detection of danger/ error),

(iii) bl44: memory functions (for acquisition of operational procedure),

(iv) b156: perceptual functions (for cognition of space), 
TABLE 1: Result of lifting experiment of robotic arms.

\begin{tabular}{lr}
\hline Robot and condition & Success rate \\
\hline iARM, standard condition & $100.0 \%(43 / 43)$ \\
iARM, without TE & $97.7 \%(42 / 43)$ \\
iARM, without TE and without TC & $95.3 \%(41 / 43)$ \\
iARM, without TE, without TC, and without RF & $93.0 \%(40 / 43)$ \\
RAPUDA, standard condition & $95.3 \%(41 / 43)$ \\
RAPUDA, without TE & $79.1 \%(34 / 43)$ \\
\hline
\end{tabular}

(v) b164: higher-level cognitive functions (for detection of danger/error),

(vi) b176: mental function of sequencing complex movements (for action planning),

(vii) b210: seeing functions (for cognition of operation interface, detection of danger/error),

(viii) b760: control of voluntary movement functions,

(a) b7600: control of simple voluntary movements (for pushing switch of operation interface).

These are minimum functions of the level of "body functions and structure" in order to make full use of the robotic arm. In addition to these, in order to prevent the disuse syndrome, the assessment by medical doctors and experts from the viewpoint, whether the assistance is excessive or not, is indispensable. It leads to the representation of (D) indication and contraindication of users.

\section{Issues on Utilization of ICF in Development Process of Assistive Robots}

Based on our experiences, we describe some issues on utilization of ICF in assistive-robots development, for further development of our framework.

4.1. Utilization of ICF Model. We have shown some practical examples of utilization of ICF in the development process of assistive robots in Section 3. We demonstrated some advantages of using ICF, but all of them are only related to one aspect of ICF as a sharable and exhaustive classification about human life (Section 2.2.1). In other words, we have only used ICF as a set of vocabularies to indicate various things.

The goal of our framework is the life design using ICF and assistive technologies. For this purpose, we must develop a methodology of analysis of the "true needs" and the "effect/impact" of assistive technologies in the users' whole life. The ICF model (Section 2.2.2) is another feature of the ICF framework providing useful viewpoints to analyze human life. We should utilize the ICF model and clarify the relations among many elements and factors of many levels of the functioning. Figure 10 shows an image of the relations to realize a goal (growth of participation). It represents how the lower level activities linked to the realization of higher-level concepts (participation).
4.2. Need for Support Tool for Utilization of ICF. ICF provides useful framework for robot development, but we also felt that ICF is somewhat hard to use by nonexperts. In fact, although ICF is utilized in various applications such as nursing care, rehabilitation, and special needs education, it is still recognized as hard to use mainly because of too many items and difficult terms [26]. In addition, Okawa et al. [27] remarked that the deep analysis and evaluation in human lives require high level of expert knowledge and clinical experiences. We therefore believe that a support tool which assists the life design will be a great help for nonexperts.

We are planning to develop a life design support tool using the ICF framework, which assists the life design shown in Figure 3. Figure 11 shows a schematic diagram of the tool. This tool accumulates a lot of knowledge about relations among many factors of ICF as functioning models. The user interactively conduct the analysis and design process using these models. This tool will be a kind of expert system using ICF.

4.3. Need for Reorganization of ICF for Utilization in Engineering Systems. When we use ICF in knowledge systems like the design support tool mentioned above, the ICF categories (vocabularies) should be sharable, reusable, and scalable. The current ICF intends to be used as a sharable language describing human life. However, ICF seems not to have in itself any mechanism facilitating the sharing. Efforts for the sharing are entrusted to the user side.

As for the reusability and the scalability, although ICF is classified into many categories, the systematization of them is not enough. Kumar and Smith [28] investigated the ICF categories and pointed out seven problems related to the systematicity. We also confirmed the problems in our study. They are digested into the following three items.

(1) Criteria of categorization are not consistent.

(2) Categorization is incomplete (segmentalization is insufficient).

(3) Subsumption (is-a relation) is overemphasized.

An example of the item (1) is shown below.

[b110: consciousness functions] is positioned into the series of concept lined by is-a relations which starts with [b: body functions] followed by [b1: mental functions]. The concept series is about "function." However, [b110: consciousness functions] has some childconcepts which are not the concept about function 


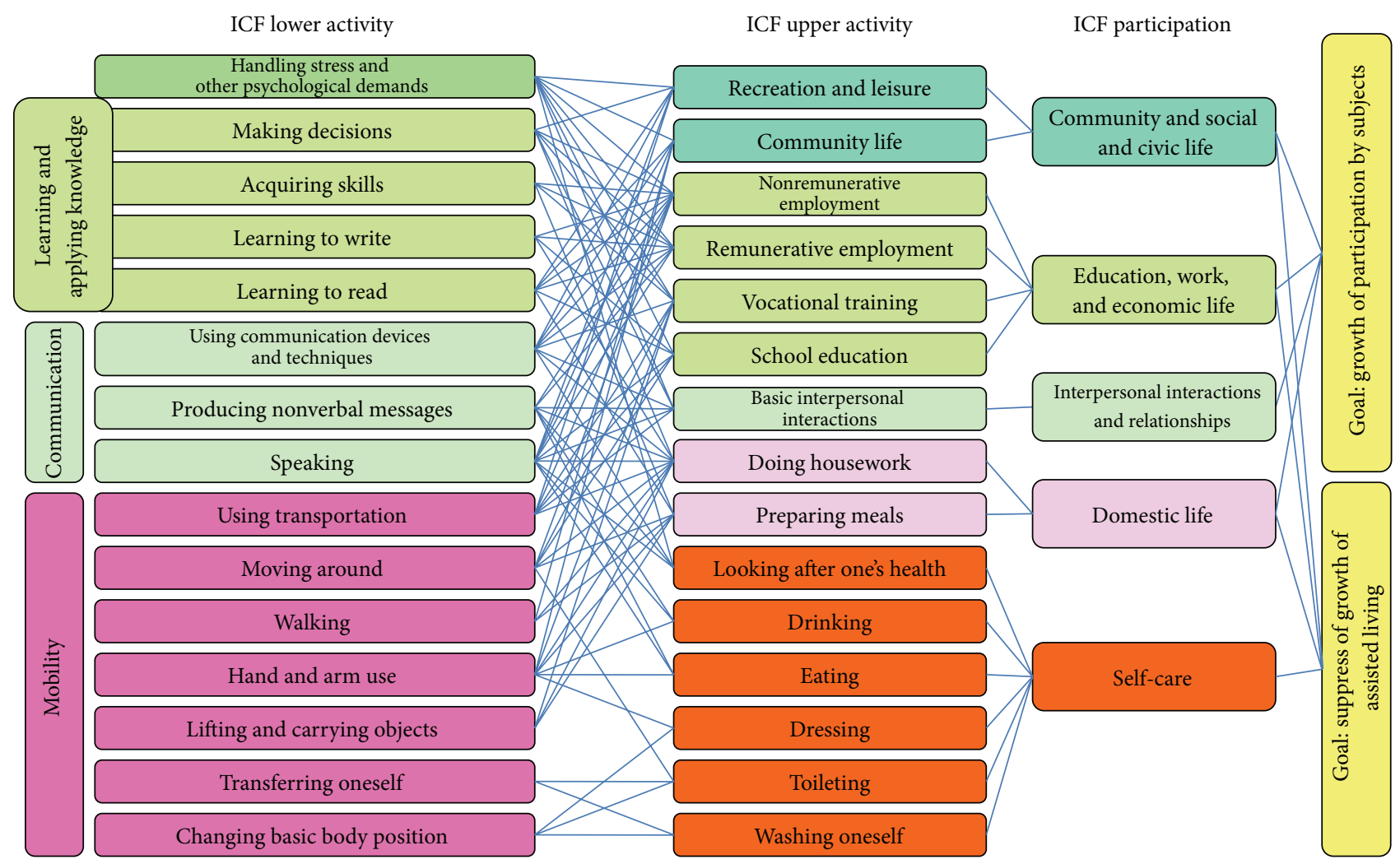

FIGURE 10: Image of relation among many factors of several levels of functioning.

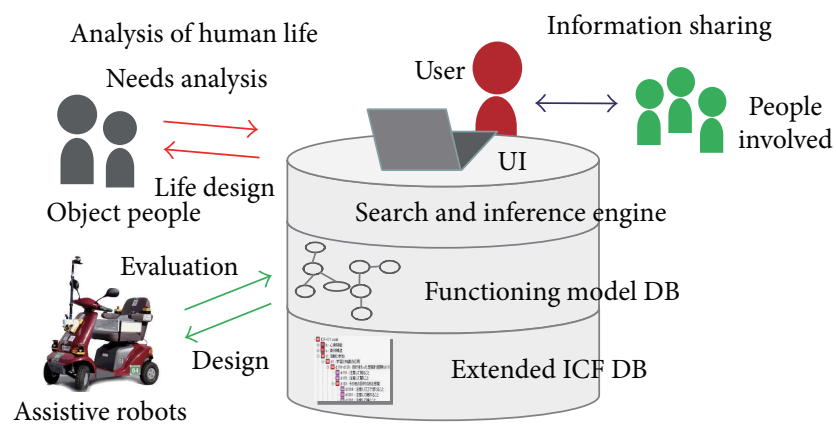

Figure 11: Life design support tool based on an extended ICF database [14].

such as [b1100: state of consciousness] and [b1101: continuity of consciousness].

Such inconsistencies are seen everywhere in ICF. Inconsistent criteria of categorization (conceptualization) make the computational semantics vague and prevent knowledge sharing, reuse, and extension. This is a fatal problem to knowledge systems. Mea and Simoncello [29] pointed out similar problems about the ICF's systematicity, from ontology engineering point of view.
Also, the vocabulary system should be readable for both human and computer. ICF is mainly written in natural language for human, not for computer. Though the ICF categories are coded by a character and a numeric code, it is not machine-readable since it has no semantic foundation.

In order to solve these issues, we started reorganization of ICF vocabularies [14] by ontology engineering approach $[20,30]$. This effort will contribute to the facilitation of ICF utilization in engineering systems like the framework of assistive-robots development we proposed.

\section{Concluding Remarks}

We proposed a framework of evaluation and design of assistive robots using ICF. We demonstrated the utility of the concept through some examples of practical application such as the analysis of daily living, the design of assistive robots, and the evaluation of assistive robots. From these experiences, we also revealed the issues for further utilization of ICF in engineering systems.

The application of ICF to the engineering field has been just started. There will be many trials and errors, but we will continue the development of the framework in order to realize the development and the popularization of "truly useful assistive robots." For this purpose, not only the robotics researchers and developers but also various types of experts, 
for example, medical doctors, healthcare workers, care managers, and the users, should work in collaboration.

\section{Conflict of Interests}

The authors declare that there is no conflict of interests regarding the publication of this paper.

\section{Acknowledgment}

The authors' deepest appreciation goes to Dr. Woo-Keun Yoon and Mr. Hiroshi Kosaka who provided some experimental results.

\section{References}

[1] S. P. Levine, D. A. Bell, L. A. Jaros, R. C. Simpson, Y. Koren, and J. Borenstein, "The Navchair assistive wheelchair navigation system," IEEE Transactions on Rehabilitation Engineering, vol. 7, no. 4, pp. 443-451, 1999.

[2] A. Tsukahara, R. Kawanishi, Y. Hasegawa, and Y. Sankai, "Sit-tostand and stand-to-sit transfer support for complete paraplegic patients with robot suit HAL," Advanced Robotics, vol. 24, no. 11, pp. 1615-1638, 2010.

[3] G. R. B. E. Römer, H. J. A. Stuyt, and A. Peters, "Cost-savings and economic benefits due to the Assistive Robotic Manipulator (ARM)," in Proceedings of the IEEE 9th International Conference on Rehabilitation Robotics (ICORR '05), pp. 201-204, Chicago, Ill, USA, July 2005.

[4] V. Maheu, J. Frappier, P. S. Archambault, and F. Routhier, "Evaluation of the JACO robotic arm: clinico-economic study for powered wheelchair users with upper-extremity disabilities," in Proceedings of the IEEE International Conference on Rehabilitation Robotics (ICORR '11), pp. 1-5, Zurich, Switzerland, July 2011.

[5] K. Wada and T. Shibata, "Living with seal robots-its sociopsychological and physiological influences on the elderly at a care house," IEEE Transactions on Robotics, vol. 23, no. 5, pp. 972980, 2007.

[6] T. Komatsu, T. Iwaoka, and M. Nambu, "Leaving a Message with the PaPeRo Robot: the Effect of Interaction Experience with Real or Virtual PaPeRo on Impression Evaluation," in Proceedings of the Entertainment Computing (ICEC '06), vol. 4161 of Lecture Notes in Computer Science, pp. 37-42, 2006.

[7] NEDO Project Index, 2012, http://www.nedo.go.jp/english/ activities_nedoprojects.html.

[8] W. M. Bortz II, “The disuse syndrome," Western Journal of Medicine, vol. 141, no. 5, pp. 691-694, 1984.

[9] International Classification of Functioning, Disability and Health (ICF), World Health Organization, Geneva, Switzerland, 2001.

[10] ICF illustration library, http://www.icfillustration.com/icfil_ eng/top.html.

[11] Y. Matsumoto, Y. Nishida, Y. Motomura, and Y. Okawa, "A concept of needs-oriented design and evaluation of assistive robots based on ICF," in Proceedings of the IEEE International Conference on Rehabilitation Robotics (ICORR '11), pp. 686-691, Zurich, Switzerland, 2011.

[12] H. Kousaka, H. Mizoguchi, M. Yoshikawa, H. Tanaka, and Y. Matsumoto, "Role analysis of dominant and non-dominant hand in daily life," in Proceedings of the IEEE International Conference on Systems, Man, and Cybernetics (SMC '13), pp. 3972-3977, Manchester, UK, 2013.
[13] E. Oyama, W. Yoon, Y. Wakita et al., "Development of evaluation indexes for assistive robots based on ICF", in Proceedings of the IEEE International Symposium on on Robot and Human Interactive Communication, pp. 221-227, Paris, France, 2012.

[14] H. Tanaka and Y. Matsumoto, "Towards reorganization of international classification of functioning, disability and health for design of assistive robots," in Proceedings of the IEEE Workshop on Advanced Robotics and its Social Impacts (ARSO '13), pp. 100105, Tokyo, Japan, 2013.

[15] T. Kato, K. Ukai, Y. Kodama, Y. Tsukumori, Y. Ando, and M. Mizukawa, "Investigation of service management system design of RT-service," in Proceedings of the IEEE International Conference on Fuzzy Systems, pp. 1496-1500, Jeju, Korea, August 2009.

[16] M. Narita and M. Shimamura, "A report on RSi (Robot Services Initiative) activities," in Proceedings of the IEEE Workshop on Advanced Robotics and its Social Impacts, pp. 265-268, Nagoya, Japan, June 2005.

[17] M. Mizukawa, H. Matsuka, T. Koyama, and A. Matsumoto, "ORiN: open robot interface for the network, a proposed standard," Industrial Robot, vol. 27, no. 5, pp. 344-350, 2000.

[18] B. E. Ainsworth, W. L. Haskell, M. C. Whitt et al., "Compendium of physical activities: an update of activity codes and MET intensities," Medicine and Science in Sports and Exercise, vol. 32, no. 9, pp. S498-S504, 2000.

[19] M. Sivan, R. J. O'Connor, S. Makower, M. Levesley, and B. Bhakta, "Systematic review of outcome measures used in the evaluation of robot-assisted upper limb exercise in stroke," Journal of Rehabilitation Medicine, vol. 43, no. 3, pp. 181-189, 2011.

[20] Y. Kitamura and R. Mizoguchi, "Ontology-based systematization of functional knowledge," Journal of Engineering Design, vol. 15, no. 4, pp. 327-351, 2004.

[21] iARM website (Exact Dynamics), http://www.exactdynamics $. n l /$ site/?page $=$ iarm.

[22] Y. Wakita, W. Yoon, and N. Yamanobe, "User evaluation to apply the robotic arm RAPUDA for an upper-limb disabilities Patient's Daily Life," in Proceedings of the IEEE International Conference on Robotics and Biomimetics (ROBIO '12), pp. 14821487, Guangzhou, China, 2012.

[23] Y. Wakita, N. Yamanobe, K. Nagata, and E. Ono, "Single-switch user interface for robot arm to help disabled people using rtmiddleware," Journal of Robotics, vol. 2011, Article ID 528425, 13 pages, 2011.

[24] Y. Choi, T. Deyle, T. Chen, and J. D. Glass, "A list of household objects for robotic retrieval prioritized by people with ALS," in Proceedings of the IEEE International Conference on Rehabilitation Robotics (ICORR '09), pp. 510-517, Kyoto, Japan, 2009.

[25] H. Nguyen and C. C. Kemp, "Bio-inspired assistive robotics: service dogs as a model for human-robot interaction and mobile manipulation," in Proceedings of the 2nd Biennial IEEE/RASEMBS International Conference on Biomedical Robotics and Biomechatronics (BioRob '08), pp. 542-549, Scottsdale, Ariz, USA, October 2008.

[26] A. Tokunaga, “Trends and perspective of the use of international classification of functioning, disability and health (ICF) on special needs education in Japan," Journal of Special Education in the Asia Pacific, vol. 2, pp. 17-30, 2006.

[27] Y. Okawa et al., "A study on evaluation method of research and development of assistive devices contributing the improvement of functioning of disabled people [in Japanese], FY2008 Report of investigation research project on services and supports 
for persons with disabilities, Ministry of Health, Labour and Welfare," pp. 40, 2009, http://www.mhlw.go.jp/bunya/shougaihoken/cyousajigyou/jiritsushien_project/seika/research_09/dl/ result/08-04a.pdf.

[28] A. Kumar and B. Smith, "The Ontology of Processes and Functions: A Study of the International Classification of Functioning, Disability and Health," Proc. AIME, 2005 workshop Biomedical Ontology Engineering, 2006, http://ontology.buffalo.edu/medo/ICF.pdf.

[29] V. D. Mea and A. Simoncello, "An ontology-based exploration of the concepts and relationships in the activities and participation component of the international classification of functioning, disability and health," Journal of Biomedical Semantics, vol. 3, article 1, 2012.

[30] R. Mizoguchi, "Knowledge acquisition and ontology," in Proceedings of the KB \& KS '93, pp. 121-128, 1993. 

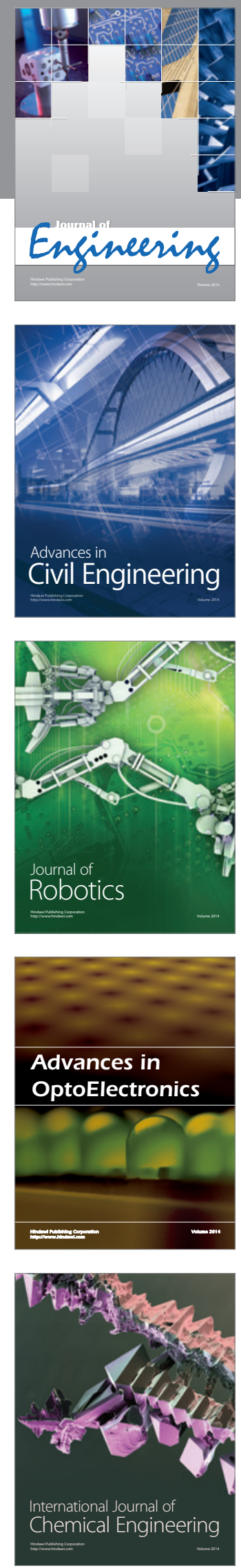

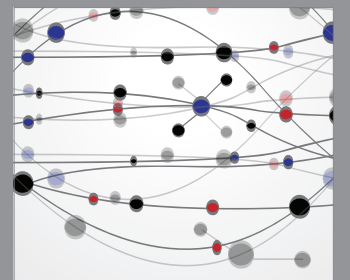

The Scientific World Journal
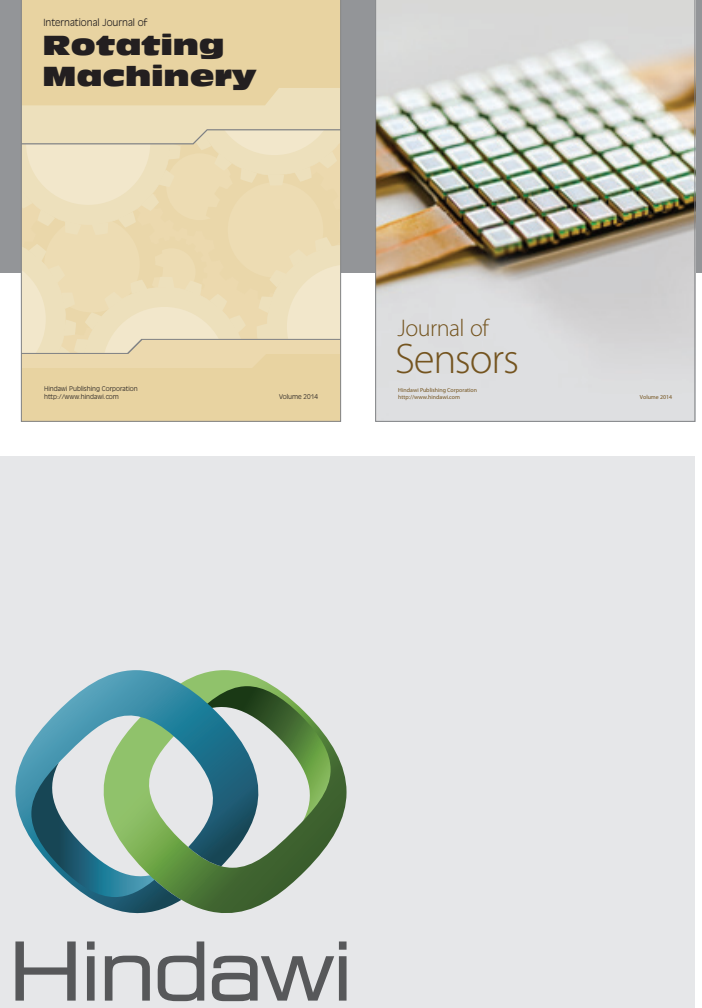

Submit your manuscripts at http://www.hindawi.com
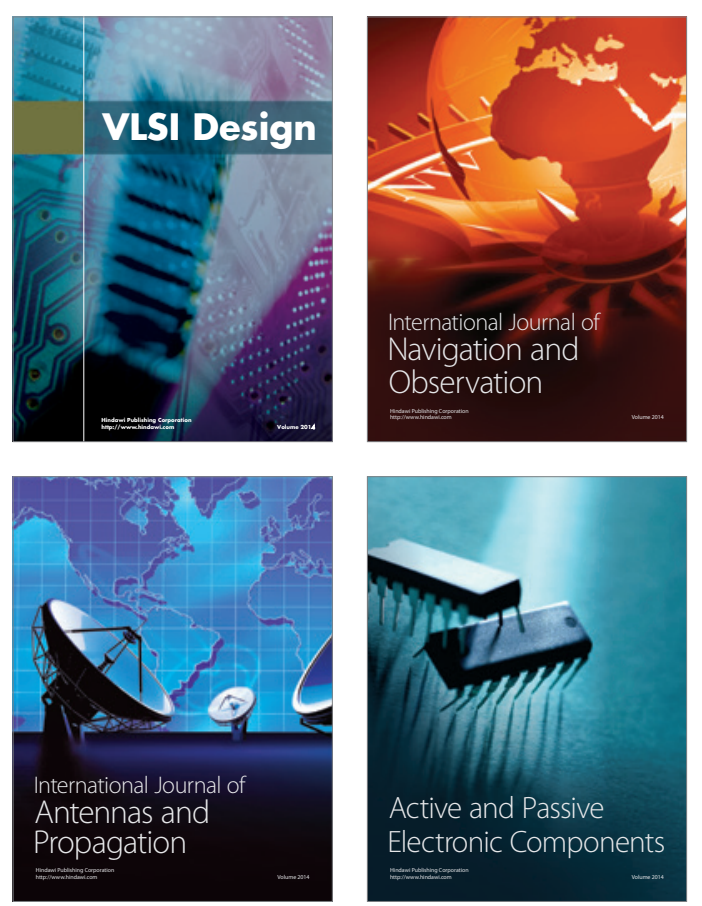
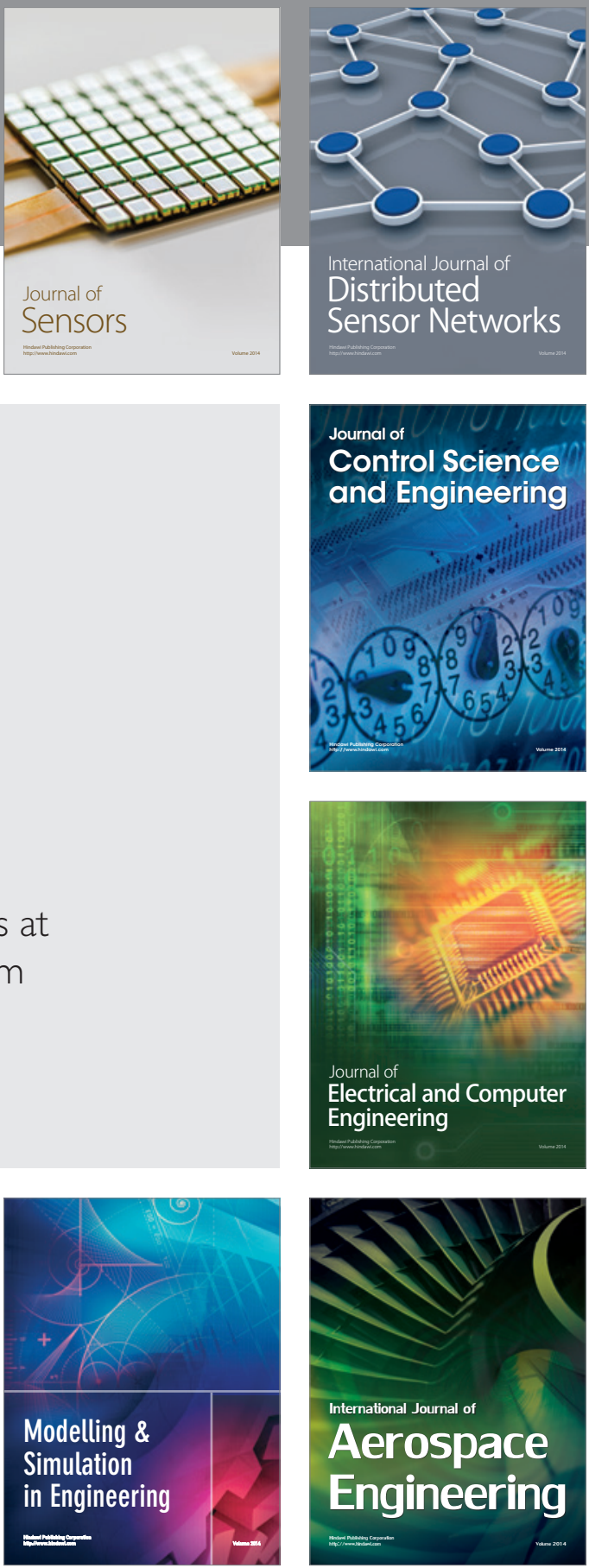

Journal of

Control Science

and Engineering
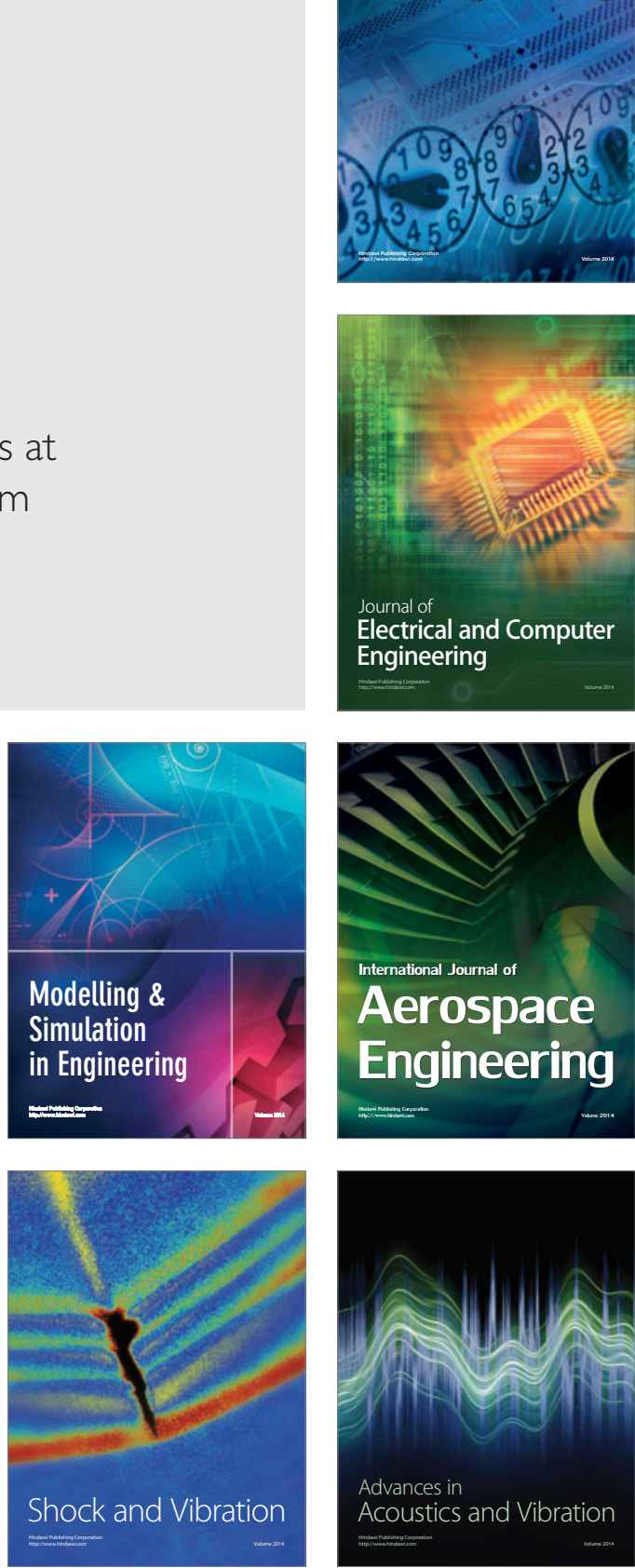